\title{
Directional solidification cells with grooves for a small partition coefficient
}

\author{
John D. Weeks and Wim van Saarloos \\ AT\&T Bell Laboratories, Murray Hill, New Jersey 07974
}

(Received 5 October 1988)

\begin{abstract}
Using the asymptotic matching procedure of Dombre and Hakim, we determine properties of the steady-state cellular structures with deep narrow grooves observed in directional solidification experiments. The method is valid in the experimentally relevant region of small partition coefficient and finite Péclet number. An extension of the Scheil equation for the grooves is given, and the importance of conservation in determining the groove closing is pointed out.
\end{abstract}

Recently there has been much progress in our understanding of the steady-state patterns formed in the directional solidification (DS) of a binary mixture. In this technologically important process, solidification occurs when a thin sample of melt is pulled at a velocity $V$ through a fixed temperature gradient. ${ }^{1}$ Small-amplitude cellular patterns have sometimes been observed as $V$ is increased, ${ }^{2}$ but more commonly the liquid-solid interface breaks up into deep fingerlike cells with narrow grooves ${ }^{3}$ that are often terminated by a bubblelike closure. ${ }^{4}$

In conceptually important work, Dombre and Hakim ${ }^{5}$ (DH) showed analytically that the steady-state equations for a simplified model of DS allowed a continuous band of cellular wavelengths, even when surface-tension effects are taken into account. In this Rapid Communication, we make further analytic progress by exploiting the small value of the partition (segregation) coefficient $k$ usually found in solidification experiments. This yields an analytic description of a class of small-amplitude cellular solutions and also leads to a generalization of the DH matching procedure for deep cells that holds for realistic values of experimental parameters. (DH considered $k=1$ and very small Péclet numbers.) Again, we find a continuous band of wavelengths. We also discuss some aspects of the bubble closure. Since there is a large parameter space, our work serves as a useful complement to recent numerical studies, ${ }^{4,6}$ where these issues are also being actively investigated.

In most of this work we use the one-sided model, ${ }^{1}$ where impurity diffusion in the solid is neglected. Since the ratio of diffusion constants in the solid and melt, $\beta \equiv D^{s} / D$, is typically much smaller than $10^{-2}$, this is usually a good approximation. However, for very deep grooves and in the reentrant part of the bubble closure the approximation must break down. This is discussed at the end of this paper.

The steady-state equations for the one-sided model of DS in two dimensions can be written ${ }^{1}$

$$
\begin{aligned}
& \nabla^{2} u+\frac{1}{l_{D}} \frac{\partial u}{\partial z}=0, \\
& {\left[u_{i}-k\left(u_{i}-1\right)\right] \cos \theta=-l_{D}(\hat{\mathbf{n}} \cdot \nabla u)_{i},} \\
& u_{i}=1-z_{i} / l_{T}-d_{0} \kappa .
\end{aligned}
$$

Here $d_{0}$ is the chemical capillary length, $l_{T}$ the thermal length, $l_{D} \equiv D / V$ a diffusion length, $\kappa$ the interface curva- ture, and $\theta$ is the angle between the interface normal $\hat{\mathbf{n}}$ and the growth direction, taken parallel to the $z$ axis. ${ }^{7} \mathrm{~A}$ subscript $i$ denotes a quantity evaluated at the interface, and the superscript $s$ denotes the solid phase. The dimensionless field $u \equiv\left(c-c_{\infty}\right) / \Delta c_{0}$ measures the impurity concentration $c$ in the liquid relative to that far from the interface, $c_{\infty}$. It is normalized by the planar miscibility gap $\Delta c_{0}=c_{0}(1-k)$, with $k$ the partition coefficient and $c_{0}$ the concentration at the planar steady-state interface. Conservation requires that $c_{\infty}=k c_{0}$.

Equation (3) takes account of surface-tension effects and imposes local equilibrium at the interface. Equation (2) expresses local conservation of impurities as the interface advances. In the one-sided model, impurity atoms incorporated into the solid at the interface undergo no fur-
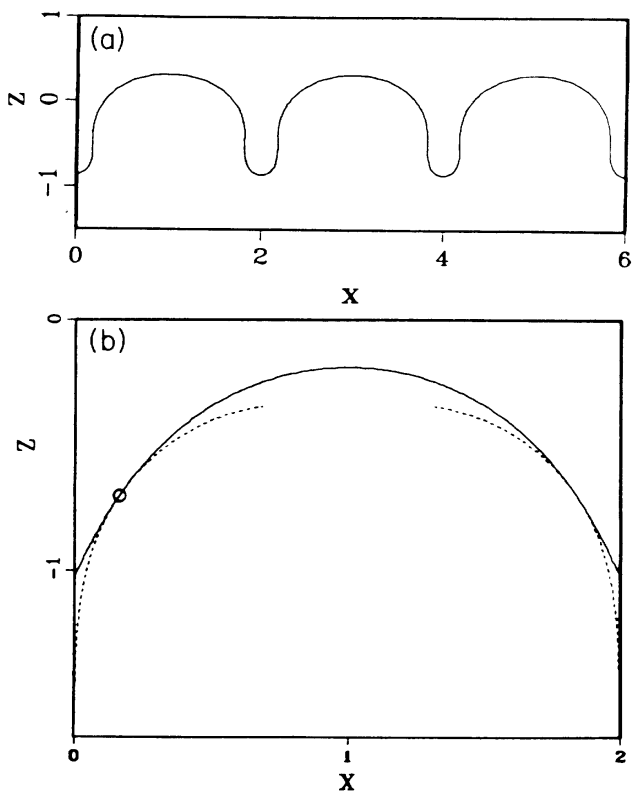

FIG. 1. (a) Finite-amplitude solution of Eq. (8) for $p=3$, $v=2, k=0.1$, and $\sigma=0.29$ with $B$ chosen to satisfy conservation condition (5). (b) The (outer) solution of Eq. (8) for $p=1$, $v=2, k=0.1, \sigma=0.78$, and $1-\lambda=0.03$ (solid line), and an associated inner solution of Eq. (9) (dashed line), determined approximately by "patching" the function and its derivative to the outer solution at the point indicated. As $\lambda \rightarrow 1$, this is essentially equivalent to the asymptotic matching procedure of $\mathrm{DH}$. 
ther diffusion. Thus, in the steady state, the condition $c_{i}^{s}=k c_{i}$ is equivalent to

$$
u^{s}(x, z)=k\left[u\left(x, z_{i}\right)-1\right],
$$

where $u\left(x, z_{i}\right)=u_{i}$ gives the concentration in the melt at the interface position $z_{i}(x)$.

We are interested in periodic cellular solutions of (1)-(3), with period $2 a$. It is customary to describe the system by the dimensionless variables $p \equiv a / l_{D}$, where $p$ is the Péclet number, and $v \equiv l_{T} / l_{D}$. The planar interface is unstable for $v \gtrsim 1$ and values of $p$ of $O(1)$ are often seen in experiments. In what follows we take $a$ as the unit of length, and measure $x$ from the center of the grooves.

For steady-state patterns, the integrated impurity flux in the $-z$ direction across any horizontal line segment extending from $x=0$ to $x=1$ must be a constant, independent of segment position $z$, since no net flux can escape through the vertical lines of symmetry at $x=0,1$ (see Fig.
1). For $z>z_{t}$, with $z_{t}$ the tip position of a pattern, a dimensionless measure of this flux is given by $F(z)$ $\equiv \int_{0}^{1} d x\left(u+p^{-1} \partial u / \partial z\right)$, where the first term is the convective flux arising from the transformation to the moving frame used in (1)-(3), and the second is the flux due to diffusion, occurring by assumption only in the liquid. Far from the interface we have $u=\partial u / \partial z=0$, so global conservation of impurities requires that $F=0$ everywhere. For $z<z_{t}$, we have similarly

$$
\begin{aligned}
F(z)=0= & \int_{0}^{x_{i}} d x\left(u+p^{-1} \partial u / \partial z\right) \\
& +k \int_{x_{i}}^{1} d x\left[u\left(x, z_{i}\right)-1\right],
\end{aligned}
$$

where we have used Eq. (4). Here $x_{i} \equiv x_{i}(z)$ and $z_{i} \equiv z_{i}(x)$ give the interface positions as functions of $z$ and $x$, respectively. Differentiating (5), we have the exact result

$$
d x_{i} / d z\left[u_{i}+p^{-1}(\partial u / \partial z)_{i}\right]+\int_{0}^{x_{i}} d x\left(\partial u / \partial z+p^{-1} \partial^{2} u / \partial z^{2}\right)-k d x_{i} / d z\left(u_{i}-1\right)=0
$$

Equation (6) immediately suggests different approximations valid near the tip and deep in the grooves for the shape of periodic cellular patterns. By use of the diffusion equation (1), Eq. (6) can be rewritten exactly as

$$
u_{i}+p^{-1}(\partial u / \partial z)_{i}-k\left(u_{i}-1\right)=p^{-1}(\partial u / \partial x)_{i}\left(d z_{i} / d x\right)
$$

which is equivalent to Eq. (2). Since both $d z_{i} / d x$ and $(\partial u / \partial x)_{i}$ must vanish by symmetry at $x=1$, the righthand side of Eq. (7) is always small near the tip. This is consistent with the (approximate) existence of a onedimensional field $u=u(z)$, which makes the right-hand side of (7) vanish identically. When this approximation is made, the solution to (7) can be written as

$$
\begin{aligned}
u_{i} & =(1-k)^{-1}\left(B e^{-p(1-k) z_{i}}-k\right) \\
& =1-p z_{i} / v-\left(d_{0} / a\right) \kappa,
\end{aligned}
$$

with $B$ a constant, on using (3). This gives a differential equation (8) for the interface shape $z_{i}(x)$, which should be accurate if the right-hand side of (7) is, in fact, small.

We will show elsewhere that this approximation is the leading-order term in a power-series expansion in $k$ for a class of finite-amplitude cellular solutions without grooves of Eqs. (1)-(3). It is easy to see why this should be so, since the one-dimensional field $u=B \exp (-p z)$ provides an exact solution of Eqs. (1) and (2) [or (7)] in the limit $k \rightarrow 0^{+}$. Thus, the above approximation becomes more and more accurate for small $k$, which is the experimentally relevant limit. In fact, the approximation may have a wider range of validity than this argument suggests, since Ungar and Brown ${ }^{8}$ found numerically very nearly onedimensional diffusion fields for finite-amplitude cellar solutions of (1)-(3) even with $k=0.4$.
Numerical solutions of (8), with $B$ fixed by conservation requirements derived from $\mathrm{Eq}$. (5), give a continuous family of finite-amplitude cellular shapes. An example is shown in Fig. 1(a). Anisotropic surface-tension effects produce only small changes. Although our steady-state analysis makes no predictions about the stability of these solutions, we presume that the small-amplitude solutions found for $V$ very near threshold are unstable, since at small $k$ there is a subcritical bifurcation away from the planar steady state. ${ }^{9}$ However, at larger $V$, Trivedi has given experimental examples of apparently stable finiteamplitude cells, ${ }^{2}$ which closely resemble that given in Fig. 1 (a).

It is clear that Eq. (8) has no solutions with $\kappa$ small and $-z_{i}$ large, as would be found in a deep groove. This is consistent with its derivation from Eq. (7), since deep grooves have $d z_{i} / d x$ very large, so that the right-hand side of (7) could not be neglected. In order to describe solutions with deep grooves, seen experimentally for small $k$ and $v-1$ of $O(1)$, we generalize the asymptotic matching procedure of DH. Equation (8) is our "outer" equation for the tip region. It reduces to the "pendulum" equation used by $\mathrm{DH}$ as $k \rightarrow 1$ and $p \rightarrow 0$ with proper rescalings.

To describe the deep narrow grooves, we return to Eq. (6). Deep in the grooves, the slope $d x_{i} / d z$ is very small, and a term such as $(\partial u / \partial z)_{i}$ in Eq. (6) can be accurately approximated by $d u_{i} / d z$, the $z$ derivative of the GibbsThomson condition (3), since

$$
d u_{i} / d z=(\partial u / \partial z)_{i}+(\partial u / \partial x)_{i}\left(d x_{i} / d z\right) .
$$

Further, for narrow grooves the terms under the integral in (6) can be replaced by their values at the interface. This yields our "inner" equation for the grooves, which can be written

$$
[\zeta-(1-k) p \sigma \kappa-\sigma d \kappa / d z] d x_{i} / d z-\sigma x_{i} d^{2} \kappa / d z^{2}-\sigma p x_{i} d \kappa / d z=p x_{i} /(v-1)
$$


This generalizes Eq. (33) of DH. Here $\zeta \equiv 1-(1$ $-k) p z /(v-1)$ is a measure of vertical distance that will be used repeatedly in this paper, and $\sigma \equiv v d_{0} l_{D} / a^{2}(v-1)$ $=\left(q_{\mathrm{ns}} a\right)^{-2}$, where $q_{\mathrm{ns}}$ is the neutral stability wave vector in the quasistationary approximation. ${ }^{1}$ Thus, for $\sigma$ of order unity the scale of the pattern is of order the neutral stability wavelength.

Very deep in the tails, terms involving the curvature can be neglected and (9) reduces to $\zeta d x_{i} / d z=p x_{i} /(v-1)$, which has the solution

$$
x_{i}(z)=A_{0}(\zeta)^{-1 /(1-k)} .
$$

This result is due to Scheil and Hunt. ${ }^{10}$ Since (9) has a wider range of validity, it would be interesting to compare its solutions to experimental groove shapes.

Experimental patterns often have narrow grooves even near the tip. In that case, the approximations leading to (9) remain valid within a "matching region," a distance $O(1)$ behind the tip position $z_{t}$, where (8) also holds. DH have shown how a globally acceptable finger solution can then be obtained by an asymptotic matching of the outer and inner solutions (8) and (9), the small parameter $(1-\lambda)$ being the width of the groove just below the matching region. Since the technical details are virtually identical to the work of $\mathrm{DH}$, we can be brief in our discussion here. Let $z_{m} \equiv z_{i}(x=0)$, where $z_{i}(x)$ is the (outer) solution to Eq. (8). Introducing the scaled inner variables $\tilde{x}_{i}$ and $\tilde{z}$ in (9) with $x_{i} \equiv(1-\lambda) \tilde{x}_{i}$ and $z-z_{m} \equiv\left[\sigma_{m}(1\right.$ $-\lambda)]^{1 / 3} \tilde{z}$, where $\sigma_{m} \equiv \sigma / \zeta_{m}$ plays the role of a renormalized surface-tension parameter, we obtain to lowest order in $1-\lambda$ [at $O(1-\lambda)^{2 / 3}$, after an integration, the same parameter-free inner matching equation analyzed by $\mathrm{DH}$ : $\left(1-d^{3} \tilde{x}_{i} / d \tilde{z}^{3}\right)=1 / \tilde{x}_{i}$. Matching its large $\tilde{z}$ behavior to the Taylor-series expansion of the outer solution (8) about $z_{m}$ when expressed in inner variables yields the matching conditions $\kappa_{m}=0$, and $\cot \theta_{m}=\gamma^{*}(1-\lambda)^{2 / 3}\left(\sigma_{m}\right)^{-1 / 3}$. Here $\gamma^{*}=3.19 \pm 0.02$ is determined numerically ${ }^{11}$ from the inner matching equation, as described by $\mathrm{DH}$.

Once the matching conditions are known, the parameter values at which cells with deep grooves exist can be obtained from the outer equation (8), following DH. The only practical difference with their work is that our outer equation (8) has to be solved numerically. The parameter $B$ in (8) is determined by imposing conservation at $z_{m}$ [cf. (5)]. Like DH, we find that for fixed parameters $p, a, v$, $k$, and $\sigma$, there is a unique solution obtained by matching the inner and outer solutions [see Fig. 1(b)]. Thus as $a$ is varied, a continuous family of solutions is generated. Note that surface-tension anistropy is not required for steady-state solutions to exist. Figure 2 gives $\sigma$ as a function of $1-\lambda$ for different values of $p$ and $k$. Conservation requirements as given by $\mathrm{Eq}$. (5) allow one to understand most trends, such as the fact that with other parameters held fixed, materials with smaller values of $k$ have narrower grooves (smaller $1-\lambda)$. A detailed analysis will be presented elsewhere. Here we conclude by discussing some properties of the groove closing at large $\zeta$.

The matching conditions and the shape of the finger near the tip are completely determined without any requirement that the limiting Scheil shapes (10) hold for all $\zeta \gg \zeta_{m}$ deep in the solid. In fact, diffusion in the solid can-

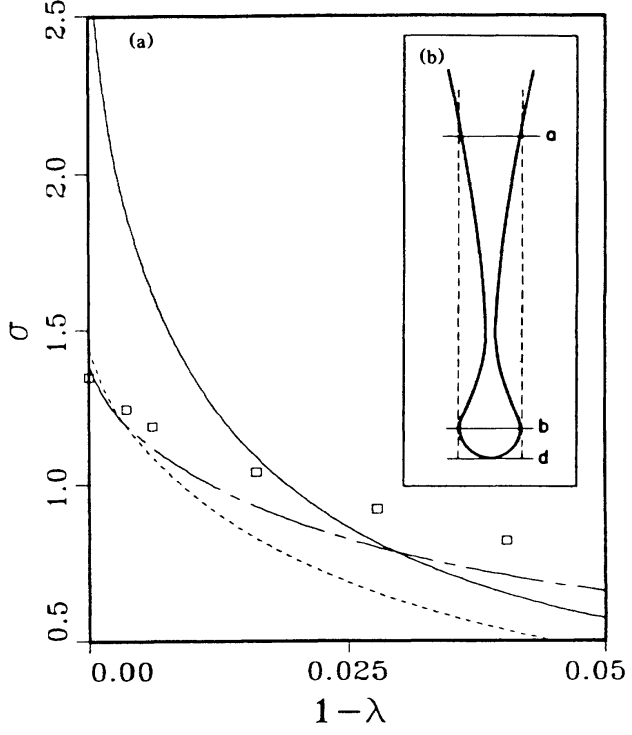

FIG. 2. (a) Variation of $\sigma$ with $1-\lambda$. The solid line gives $p=1, v=2$, and $k=0.1$. The chain-dashed line gives $p=0.01$, $v=2, k=0.95$, and is essentially identical to that given by the method of DH, where $k=1$ and $p \rightarrow 0$. Data points represent a numerical solution of the Saffman-Taylor equations by McLean (Ref. 14), to which the DH method should also apply. The dashed line gives $p=0.01, v=2$, and $k=0.1$. (b) Schematic drawing of the lower part of a groove with a bubble closure.

not be ignored when the grooves become sufficiently steep and narrow, and the predictions of Eq. (10) must then be modified. To estimate $\zeta_{c}$, the breakdown distance, note that the term $\left(D^{s} / V\right)\left(\hat{\mathbf{n}} \cdot \nabla u^{s}\right)_{i}$ should be added to the right-hand side of (2) to take account of diffusion in the solid. Using (4) to estimate $\nabla u^{s}$ (valid for $\zeta \lesssim \zeta_{c}$ and $\beta \equiv D^{s} / D$ small), we see from Eq. (2) that an additional term $-(\beta k / p)(\partial u / \partial z)_{i}\left(d z_{i} / d x\right)^{2}$, should then be added to the right-hand side of Eq. (7). Since $d z_{i} / d x=\tan \theta$ becomes arbitrarily large as $\zeta \rightarrow \infty$ according to (10), eventually this term must become significant for any $\beta>0$. We thus expect a breakdown of the Scheil equation when the neglected term becomes about equal to the left-hand side of Eq. (7), i.e., the order of the terms kept in deriving the Scheil equation. If we ignore small curvature corrections, this criterion ${ }^{12}$ gives $\beta k \approx(v-1) \zeta_{c} \cot ^{2} \theta_{c}$. For very small $\beta$, the grooves can be nearly vertical before the assumptions of the one-sided model break down.

Experiments and computer simulations have shown that the grooves often terminate at a finite $\zeta=\zeta_{b}$ with a small bubblelike closure. ${ }^{2-4}$ We outline here an approximate treatment of the closure, based on conservation requirements, that determines the order-of-magnitude scaling of most features. We assume that $\beta$ and $k$ are small, and that the maximum half width $\Delta$ of the bubble at $\zeta_{b}$ is small enough that $p \Delta \ll 1$ [see Fig. 2(b)]. It is then reasonable to replace the diffusion equation by the Laplace equation in the lower part of the bubble and assume the existence of a linear concentration gradient between $b$ and $d$, just as in the approach of DH. Combined with Eq. (3), this gives the pendulum equation analyzed by $\mathrm{DH}$. 
Using the pendulum solutions, and equating the impurity flux at $b$ to that found in the solid at $d$ then yields to lowest order $\zeta_{b}=c_{1} \sigma / \Delta^{2}+O(\Delta)$, where $c_{1}$ is a constant of $O(1)$ whose value could, in principle, be determined by matching. ${ }^{13}$

We can obtain another relation between $\zeta_{b}$ and $\Delta$ by requiring that $x_{i}\left(z_{a}\right)=\Delta$ and that the impurity flux across the groove at $z_{a}$ equals that in the solid at $z_{d}$ [see Fig. $2(\mathrm{~b})]$. The latter gives to dominant order $\zeta_{a}=k \zeta_{b}$. Finally, in the simplest case where $\Delta \lesssim 1-\lambda$, we can use the Scheil equation (10) to give an order-of-magnitude estimate of $\Delta=x_{i}\left(\zeta_{a}\right)$. This yields $\Delta /(1-\lambda)=\left(\zeta_{m} /\right.$ $\left.k \zeta_{b}\right)^{1 /(1-k)}$, where we have estimated the value of the constant $A_{0}$ by requiring that (10) gives $1-\lambda$ for $\zeta=\zeta_{m}$. Combining these results, we find

$$
\Delta /(1-\lambda)=\left[c_{1} k \sigma_{m} /(1-\lambda)^{2}\right]^{1 /(1+k)} .
$$

Note also that (10) evaluated at $\zeta_{b}$ provides an estimate for the half width $\delta$ of the narrow neck just above the bubble. This gives $\delta / \Delta=k^{1 /(1-k)}$. Thus we predict deeper grooves with a smaller ratio of neck to maximum bubble width as $k$ is decreased. These simple expressions for $\Delta, \delta$, and $\zeta_{b}$ have been derived relying mainly on conservation requirements and the assumption of local equilibrium. A quantitative analysis of experimental data could provide an important test of this basic approach.

We are grateful to D. A. Kessler and H. Levine for a helpful discussion on the breakdown of the one-sided model, to J. W. McLean for providing us with his unpublished data on Saffman-Taylor fingers, and to V. Hakim for helpful correspondence.
${ }^{1}$ For a general review, see J. S. Langer, Rev. Mod. Phys. 52, 1 (1980).

${ }^{2}$ R. Trivedi, Metall. Trans. A 15, 977 (1984); M. A. Eshelman, V. Seetharaman, and R. Trivedi, Acta. Metall. 36, 1165 (1988).

${ }^{3}$ For recent experiments, see S. de Cheveigne, G. Guthmann, and M. M. Lebrun, J. Phys. (Paris) 47, 2095 (1986); J. Bechhoefer and A. Libchaber, Phys. Rev. B 35, 1393 (1986).

${ }^{4}$ Numerical calculations that show steady-state grooved patterns with bubblelike closures include (a) L. Ungar and R. A. Brown, Phys. Rev. B 31, 5931 (1985); N. Ramprasad, M. J. Bennet, and R. A. Brown, ibid. 38, 583 (1988); (b) D. A. Kessler and $\mathbf{H}$. Levine (unpublished).

${ }^{5}$ T. Dombre and V. Hakim, Phys. Rev. A 36, 2811 (1987); see also M. Ben-Amar, T. Dombre, and V. Hakim, in Propagation in Systems Far from Equilibrium, edited by J. E. Wesfreid et al. (Spring-Verlag, New York, 1988), p. 35.

${ }^{6}$ M. Ben-Amar and B. Moussallam, Phys. Rev. Lett. 60, 317 (1988).

${ }^{7}$ We follow Langer (Ref. 1) and DH (Ref. 5) in our definitions for $l_{T}, d_{0}$, and the normalization for $u$.

${ }^{8}$ L. Ungar and R. A. Brown, Phys. Rev. B 29, 1367 (1984).

${ }^{9}$ B. Caroli, C. Caroli, and B. Roulet, J. Phys. (Paris) 48, 1767 (1982).

${ }^{10} \mathrm{~A}$ discussion is given by J. D. Hunt, Solidification and Casting of Metals (Metals Society, London, 1979). In the limit $k \rightarrow 1$, Eq. (10) reduces to the exponential profile $x_{i}=A_{0}$ $\times \exp [-p z /(v-1)]$ derived by A. Karma, Phys. Rev. Lett. 57, 858 (1986).

${ }^{11}$ Both the value for $\gamma^{*}$ given in Ref. 5 and our initial estimate were inaccurate. Both groups now agree with the result given here. Note also that we have followed DH in our definition of $p$. A consistent use of this definition requires that the unit of length is $a$ rather than $2 a$ (as is claimed by DH) in their Eqs. (28), and that the numerical values they quote for $\sigma$ be multiplied by a factor of 4 . An alternative convention, consistent with $2 a$ as the unit of length and actually intended by DH [V. Hakim (private communication)], is the use of a new Péclet number $P_{\mathrm{DH}}=2 a V / D=2 p$ and a $\sigma_{\mathrm{DH}}=\sigma / 4$.

${ }^{12}$ This estimate, valid for small $\beta$, is complementary to that of Kessler and Levine [Ref. 4(b)], who argue that $x_{c} \approx k$. However, they assume that the impurity distribution in the solid near the groove closing has a simple quadratic dependence on $x$, and hence that these impurities have been able to diffuse a distance of order the cell width. This assumption is valid only for large $\beta \gtrsim p / \Delta z$, with $\Delta z$ the groove depth.

${ }^{13}$ In principle, the pendulum solutions could be matched to the "inner" modes calculated from Eq. (9), which now grow away from the neck of the groove just above the bubble as $\zeta$ increases. This approach is complicated by the fact that the one-sided model must break down above the reentrant part of the bubble in a thin boundary layer region whose width is of order $\beta / p$, as we will discuss elsewhere. The treatment given here based on conservation makes no assumptions about the reentrant part of the bubble, and requires only that there is little impurity diffusion across the dashed vertical lines in Fig. 2(b). This should be accurate if the boundary layer region where the one-sided model breaks down is small.

${ }^{14}$ J. W. McLean and P. G. Saffman, J. Fluid Mech. 102, 455 (1981); J. W. McLean (unpublished). 